\title{
Detection of red line asymmetries in LHS 2034^
}

\author{
B. Fuhrmeister, J. H. M. M. Schmitt, and P. H. Hauschildt
}

\begin{abstract}
Hamburger Sternwarte, University of Hamburg, Gojenbergsweg 112, 21029 Hamburg, Germany e-mail: bfuhrmeister@hs.uni-hamburg.de
\end{abstract}

Received 10 December 2004 / Accepted 30 January 2005

\begin{abstract}
We report very pronounced line asymmetries during a long duration flare on the dM6 star LHS 2034 (AZ Cnc). While all lines of the Balmer series and all strong He I lines show these asymmetries, the metal lines do not. This can be explained with the help of PHOENIX model chromospheres considering the formation depth of the lines involved. Moreover, the asymmetries persist over about one hour changing shape and amplitude. Fitting the asymmetries with an additional broad Gaussian component leads us to the scenario of a series of downward propagating condensations that decelerate due to the higher density of the lower chromosphere. In addition, similar but weaker line asymmetries were found in LHS 2397a.
\end{abstract}

Key words. stars: activity - stars: flare - stars: late-type

\section{Introduction}

Stellar flares release large amounts of energy during a short time interval over a wide range of the electromagnetic spectrum. This energy is believed to result from free magnetic energy stored in the magnetic field configuration of the star and released by reconnection of magnetic field lines. Flaring is a commonly observed phenomenon in late-type stars, especially in M dwarfs, RS CVn systems, and young stars. Some dMe stars are known to increase their X-ray flux by a factor of 100 or more during flares (e.g. Favata et al. 2000). At X-ray wavelengths, energy releases in excess of $10^{37}$ erg have been reported, e.g. for the T Tauri star Par 1724 (Preibisch et al. 1995), for Algol B (Schmitt \& Favata 1999), and for the RS CVn system CF Tuc (Kürster \& Schmitt 1996). While flares on more luminous stars are typically observed at UV and X-ray wavelengths, flares on dMe stars can also be observed easily at optical wavelengths (Pettersen 1991).

A flare represents a complex magnetohydrodynamic and radiative phenomenon involving large scale plasma motions. It is therefore not surprising that for some flare events on latetype stars line asymmetries have been detected. For example, Doyle et al. (1988) found broadened wings for the lines of the Balmer series during a flare of short duration on the dM4.5e star YZ CMi. While the $\mathrm{H}_{\delta}$ and $\mathrm{H}_{\gamma}$ lines showed symmetrically broadened profiles during the flare maximum, the higher lines $\mathrm{H}_{\zeta}$ and $\mathrm{H}_{\eta}$ showed flux enhancements in the red wing of the line profiles. Doyle et al. tried to fit these broad profiles with Voigt profiles and found that even two Voigt profiles would not provide acceptable fits to the shape of the line core and the wings. In contrast, two Gaussian components were found to fit

* Based on observations collected at the European Southern Observatory, Paranal, Chile, 68.D-0166A. the profiles quite well. In the dM3.5e star AD Leo, Houdebine et al. (1993) also found red asymmetries in the core and in the wings of Balmer lines during a flare, which they interpreted as evidence of chromospheric downward condensations (CDC) similar to those seen on the Sun. CDCs originate in rapid evaporations of the pre-flare chromosphere which drive shocks upward and downward. The downward propagating shock then forms a condensation in its wake (Canfield et al. 1990). An example of asymmetries in the blue part of the wing was found in AT Mic by (Gunn et al. 1994) for the Balmer lines, as well as in the $\mathrm{Ca}$ II $\mathrm{H}$ and $\mathrm{K}$ line. These asymmetries were interpreted as upmoving material that has been heated by a particle beam from the apex of a magnetic loop.

More recently Montes et al. (1999) found line asymmetries during a long duration flare on the single, young, rapidly rotating K2 dwarf LQ Hya. Combining optical and IUE data, Montes et al. found broad wings in the $\mathrm{Mg}$ II lines and in the profiles of $\mathrm{H}_{\alpha}, \mathrm{H}_{\beta}$, He I $\mathrm{D}_{3}$ line, and $\mathrm{He} \mathrm{I} \lambda 6678$, after subtraction of a quiescent template spectrum. They fitted the lines using two Gaussian components, resulting in a blueshift of the broad line component in the impulsive phase and a redshift of the broad component in the gradual phase with the shift increasing with time. They attribute these broad components to turbulence or to upward and downward mass motions. Similar broad components of emission lines have been found by Pagano et al. (2000) in AU Mic outside of flares in FUV transitions region lines, while the chromospheric lines do not show these asymmetric redshifted broad components. The origin of the broad component is identified as microflaring.

LHS 2034 (also known as AZ Cnc) is a dM6 flare star known as an $\mathrm{X}$-ray emitter with $\log L_{\mathrm{X}} \approx 27.40$ during quiescence (Fleming et al. 1993). During the ROSAT all-sky observations it was caught in a long duration flare lasting for more 
Table 1. Exposure time, date, and UT of the beginning of each exposure of LHS 2034.

\begin{tabular}{cccc}
\hline \hline Spectrum & Date & UT & $\begin{array}{c}\text { Exposure } \\
\text { time [s] }\end{array}$ \\
\hline 1st & March, 14th & $01: 18: 40.0$ & 1200 \\
2nd & March, 14th & $01: 39: 45.0$ & 1200 \\
3rd & March, 14th & $02: 00: 42.0$ & 1200 \\
4th & March, 14th & $02: 22: 33.0$ & 600 \\
5th & March, 14th & $02: 34: 05.0$ & 600 \\
6th & March, 14th & $02: 45: 36.0$ & 600 \\
\hline 1th & March, 16th & $00: 54: 32.0$ & 1200 \\
2th & March, 16th & $01: 15: 30.0$ & 1200 \\
\hline
\end{tabular}

than three hours, possibly similar to the flare that is our subject here.

We report very strong wing asymmetries during a long duration flare on LHS 2034 and similar asymmetries in the spectrum of LHS 2397a, which are not, however, connected with a strong flare. In Sect. 2 we describe our observations, data analysis and the chromospheric models. In Sect. 3 we report on the radial and rotational velocity. In Sect. 4 we report on the emission line behavior during the long duration flare on LHS 2034. We focus on asymmetries seen comparing flaring and quiescent state. In Sect. 5 we present the results of chromosphere modelling for the decay phase of the flare on LHS 2034. A general discussion that includes a search for similar asymmetries in other stars and the weak asymmetries found in LHS 2397a and our conclusions, can be found in Sect. 6.

\section{Observations and data analysis}

The M 6 dwarf LHS 2034 was observed with ESO's Kueyen telescope on Paranal equipped with the Ultraviolet-Visual Echelle Spectrograph (UVES) on March 14th and 16th, 2002 (program ID 68.D-0166A). LHS 2034 was observed for $1.5 \mathrm{~h}$ on March 14th and for 40 min on March 16th. A large flare occurred during the observations on March 14th. Details on the observation are listed in Table 1; the instrument setup and data reduction are described in detail by Fuhrmeister et al. (2004b). The spectra were taken in dichroic mode; as a consequence, our setup covers neither the Balmer lines from $\mathrm{H}_{3}$ up to $\mathrm{H}_{8}$ nor the $\mathrm{Ca}$ II $\mathrm{H}$ and $\mathrm{K}$ lines. The typical resolution of our spectra is $\sim 45000$.

The data were reduced using IRAF in a standard way. The wavelength calibration was carried out with ThAr spectra giving an accuracy of $\sim 0.03 \AA$ in the blue arm and $\sim 0.05 \AA$ in the red arm. The data was not corrected for night sky emission lines or telluric lines. In addition to the UVES spectra there are photometric data from the UVES exposuremeter, actually taken for engineering purposes and therefore not flux calibrated. Nevertheless this data can be used in a qualitative way for assessing flares.

Spectral line fitting was done with the CORA program (Ness \& Wichmann 2002), kindly provided to us by Dr. J.-U. Ness and originally developed for analyzing high resolution X-ray spectra. The fit algorithms employed by CORA are also well suited to modelling all types of emission lines with

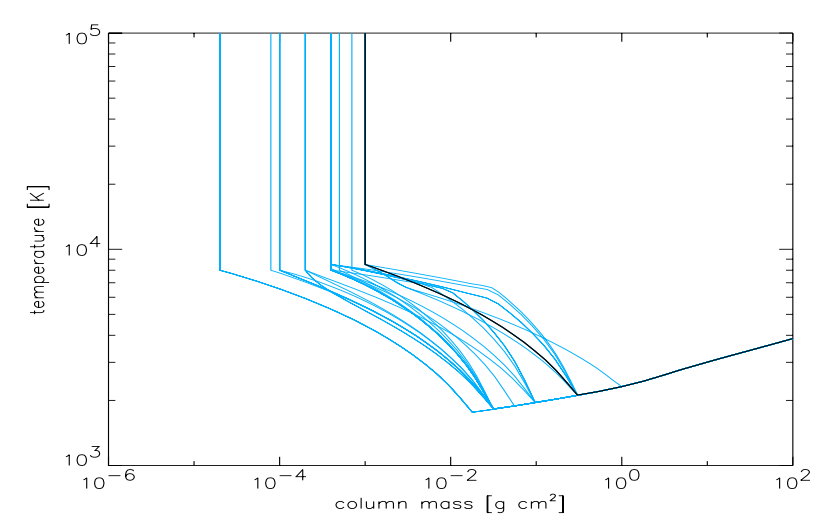

Fig. 1. Temperature vs. column mass distribution for the flare models on a logarithmic scale. The black line denotes the best fit model out of the grid.

count statistics. CORA uses a maximum likelihood method for fitting line profiles. The program also provides accurate error analysis.

In addition to line fitting with CORA we modelled the flaring atmosphere of LHS 2034 with the PHOENIX atmosphere code (Hauschildt et al. 1999). These models consist of (i) an underlying photosphere; (ii) a linear temperature rise vs. log column mass in the chromosphere; and (iii) transition region (TR) with different gradients. For the underlying photosphere, a model with $T_{\text {eff }}=2800 \mathrm{~K}, \log g=5.0$, and solar chemical composition was used. The best fit of the photosphere was determined with an $\chi^{2}$ technique using a model grid with $T_{\text {eff }}=$ $2300-3700 \mathrm{~K}$ and $\log g=4.0-5.5$ in steps of $\Delta T_{\text {eff }}=100 \mathrm{~K}$, and $\Delta \log g=0.5$. We build the flare model spectra as a linear combination of the quiescent chromospheric spectra and the flaring chromospheric spectra, similar to the approach of Mauas \& Falchi (1996). For the quiescent chromosphere, we use the last spectrum taken in the flare series, since the star is again in a more active state during our second time series. For the flaring models, we computed a grid of 42 models with the onset of the transition region at rather high pressure. The temperature vs. column mass distributions for our flare models are plotted in Fig. 1. Normally we treat only $\mathrm{H}, \mathrm{He}$, and $\mathrm{Na}$ I-IV in NLTE, but for the best fitting models we used a larger NLTE set. These models treat H, He, C I-III, N I-III, O I-III, Fe I-IV, Ti I-Ti II, Na I-IV, and Mg I-III in NLTE. All NLTE calculations take all those levels into account from either the Kurucz database (Kurucz \& Bell 1995) or from the CHIANTI database (Young et al. 2003) for He. A detailed discussion of the model construction is given by Fuhrmeister et al. (2004a).

\section{Radial and rotational velocity}

We measured the radial velocity of LHS 2034 as $64.2 \pm$ $0.6 \mathrm{~km} \mathrm{~s}^{-1}$ by determing the peak position of the crosscorrelation function between the spectrum of LHS 2034 and radial velocity template spectrum of CN Leo. For CN Leo (also known as Gl 406) we determined a radial velocity of $18.3 \pm$ $0.8 \mathrm{~km} \mathrm{~s}^{-1}$ by comparison to an absolute wavelength scale in good agreement with $19.0 \pm 1.0 \mathrm{~km} \mathrm{~s}^{-1}$ found by Mohanty \& Basri (2003). Our radial velocity for LHS 2034 agrees well 


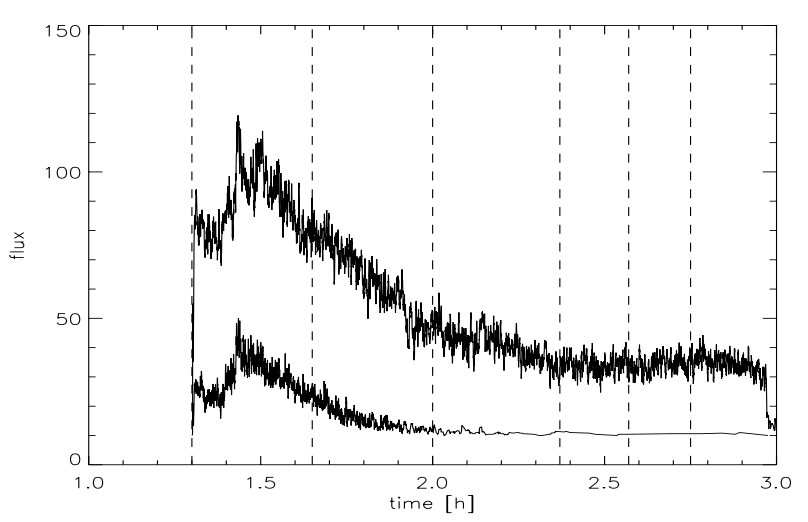

Fig. 2. Light curve of LHS 2034 during the observation on March 14th, 2002. The upper light curve corresponds to the red part of the spectrum, while the lower light curve corresponds to the blue part. The flare can be seen clearly in both spectral ranges. The vertical dashed lines indicate the beginning of each exposure. The flare ends at about the beginning of the third exposure.

with the $69.8 \pm 10.0 \mathrm{~km} \mathrm{~s}^{-1}$ determined by Reid et al. (1995). No wavelength shifts in the emission lines occur in any of our spectra of LHS 2034.

Since the radial velocity of LHS 2034 is unusually high, we also analyzed its galactic motion. According to its space velocities $U=-60.6 \mathrm{~km} \mathrm{~s}^{-1}, V=-44.3 \mathrm{~km} \mathrm{~s}^{-1}$, and $W=$ $-8.3 \mathrm{~km} \mathrm{~s}^{-1}$ (relative to the local standard of rest), it belongs kinematically to the old disk applying the criteria listed by Leggett (1992). Given the activity of LHS 2034, one expects a much younger star; however, there is no young cluster or star forming region that LHS 2034 can be attributed to in any obvious way.

We measured the rotational velocity of LHS 2034 as $v \sin (i)$ of $7.9 \pm 2.8 \mathrm{~km} \mathrm{~s}^{-1}$ again using $\mathrm{CN}$ Leo as template (Fuhrmeister et al. 2004b). Since an estimate of the rotational velocity out of the $F W H M$ of some of the emission lines leads to the same value, the emission seems to originate in a substantial area of the stellar surface, and not in just a few isolated regions.

\section{Line asymmetries on LHS 2034}

\subsection{The long duration flare on LHS 2034}

The optical light curve of the observation on 14th March 2002 shows a major long duration flare as seen in Fig. 2. We have no coverage of the onset of the flare, but we do cover the decay phase; and in the last three spectra the star is probably back to its "quiescent emission". During the flare hundreds of emission lines appear, most of them decaying completely in the course of the first three spectra. Very few absorption lines are seen in the spectra.

The HeI lines found in the flare spectrum is listed in Table 2. For further analysis we only use the $\mathrm{He}_{\mathrm{ID}} \mathrm{D}_{3}$ line at $5875 \AA$, and the He I singlets at 4921 and $5015 \AA$, since they are the lines with the most obvious asymmetry. The $\mathrm{HeID}_{3}$ transition is a multiplet consisting of six component lines at wavelengths $5875.60,5875.614,5875.615,5875.63,5875.64$, and $5875.97 \AA$, respectively. The first five components are too
Table 2. List of identified He I lines in the first spectrum of LHS 2034. Column one gives the rest wavelength after Moore (1972), Col. 2 gives an indication of the level of asymmetry, and finally column three gives further comments.

\begin{tabular}{ccc}
\hline \hline Wavelength $[\AA]$ & Asymmetry & Comment \\
\hline 3187.743 & very weak & weak line \\
3554.394 & blended & very weak line \\
3613.641 & blended & very weak line \\
3819.606 & very weak & very weak line \\
4921.931 & detected & \\
5015.675 & detected & \\
5875.650 & detected & two line components \\
\hline
\end{tabular}

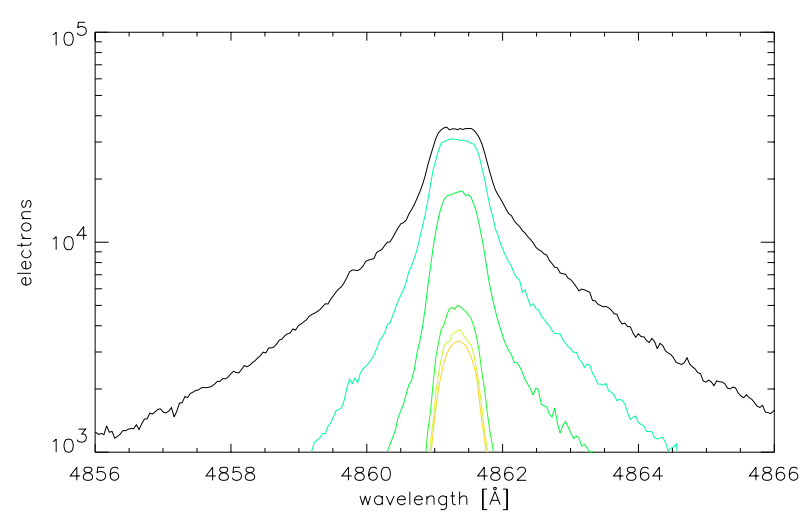

Fig. 3. Spectral sequence of the $\mathrm{H}_{\beta}$ line on a logarithmic plot. Note the large asymmetries in the second and third spectra: in the red wing there is considerably more flux than in the blue.

close together to be resolved in the spectra, but the last component can be resolved and is actually seen in the spectra.

In the first flare spectrum, the Balmer series is clearly seen up to $\mathrm{H}_{18}$, while in the fifth and sixth spectrum $\mathrm{H}_{11}$ is the highest detected Balmer line. For the $\mathrm{H}_{\alpha}$ line in the first (flare) spectrum we determine an equivalent width $(E W)$ of $109 \AA$, and for the $\mathrm{H}_{\beta}$ line we find an $E W$ of $149 \AA$. The $\mathrm{H}_{\beta}$ line is shown in Fig. 3, while an example of the $\mathrm{H}_{\alpha}$ line can be found in Fig. 6.

\subsection{Line symmetries and asymmetries on LHS 2034}

An example of line asymmetries for the $\mathrm{H}_{\beta}$ line is shown in Fig. 3. The $\mathrm{H}_{\beta}$ line displays very broad wings in the first three spectra of the time series during the flare and no significant wings in the last three spectra taken during the constant part of the lightcurve. These unbroadened lines are highly symmetrical at first glance, while the broadened lines show additional flux in the red wings leading to much shallower slopes on the red side than on the blue side. The line fitting procedure also revealed small asymmetries in the last three spectra.

The shapes of the He lines and of the lines of the Balmer series have to be contrasted with the line shapes of metal lines that remain unbroadened. This difference is exemplified in Fig. 4, where we compare the $\mathrm{He}_{\mathrm{I}} \mathrm{D}_{3}$ line and the $\mathrm{Na} \mathrm{D}$ lines. Obviously, the $\mathrm{Na} \mathrm{D}$ lines are highly symmetrical, while the $\mathrm{He}$ lines are not. 


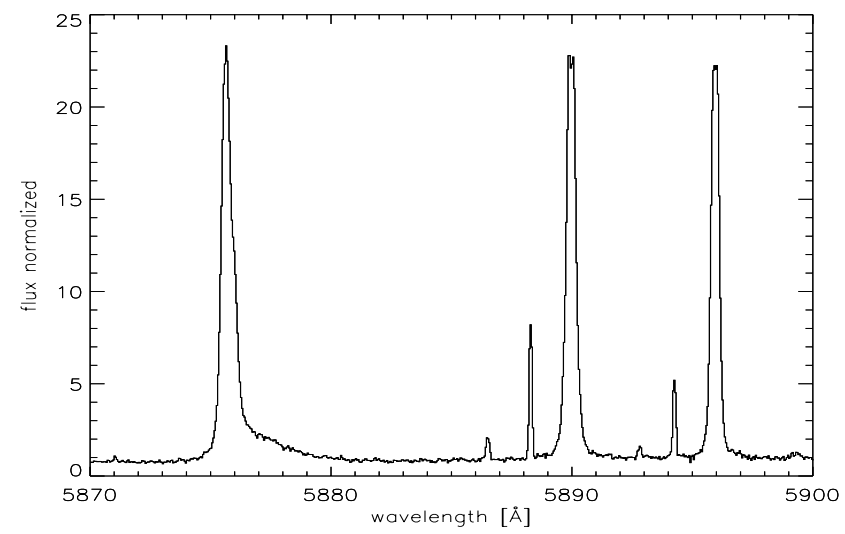

Fig. 4. Spectral wavelength around the $\mathrm{He} \mathrm{ID}_{3}$ line at $5875 \AA$ and the $\mathrm{NaD}$ lines at 5890 and $5896 \AA$ in the second spectrum of the series. Though the strength of the line is about the same, the $\mathrm{He} \mathrm{ID}_{3}$ line shows red wing asymmetry while the Na D lines does not. All other (narrow) emission lines seen in this part of the spectrum are known airglow lines.

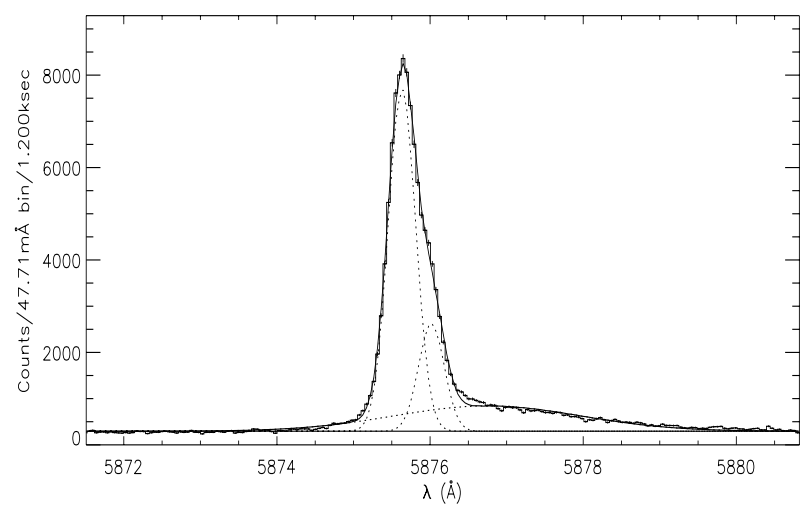

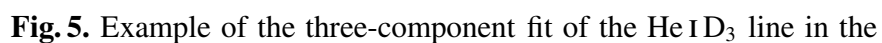
second spectrum. Plotted are all three components, the resulting fit, and the data.

In order to quantitatively describe the measured line profiles we modelled the lines with up to three Gaussian components, for each we fitted the amplitude, the Gaussian $\sigma$, and the central wavelength as free parameters of each component. An example of the general fit quality is given in Fig. 5 for the $\mathrm{He} \mathrm{ID}_{3}$ line. Despite the low signal the quality of the fits of the high Balmer lines and blue He I lines are of comparable quality. Fits with one and two Gaussians clearly show the need for a two Gaussian fit. For example for the first spectrum and the $\mathrm{H}_{\beta}$ line a one-component fit resulted in $\chi^{2}$ of 568 , while a twocomponent fit resulted in $\chi^{2}$ of 29 (the line is fitted rather poorly since it suffers from some self-absorption). For the $\mathrm{H}_{11}$ line with a much lower $S / N$, a one-component fit resulted in $\chi^{2}$ of 2.76, while two components lead to $\chi^{2}=0.66$. We did encounter difficulties in fitting the $\mathrm{H}_{\alpha}$ line, since it shows strong self-absorption in the line center. The peak of the Gaussian plays a large role in the fitting process so that the fit is severely hampered by self-absorption. We thus decided to exclude the data in the line center from the fitting process and fitted the amplitude of the narrow component manually. This procedure resulted in satisfactory fits as illustrated in Fig. 6. Since for the narrow component neither the wings nor the peak but only the side lobes are used for the fit, the Gaussian cannot be well

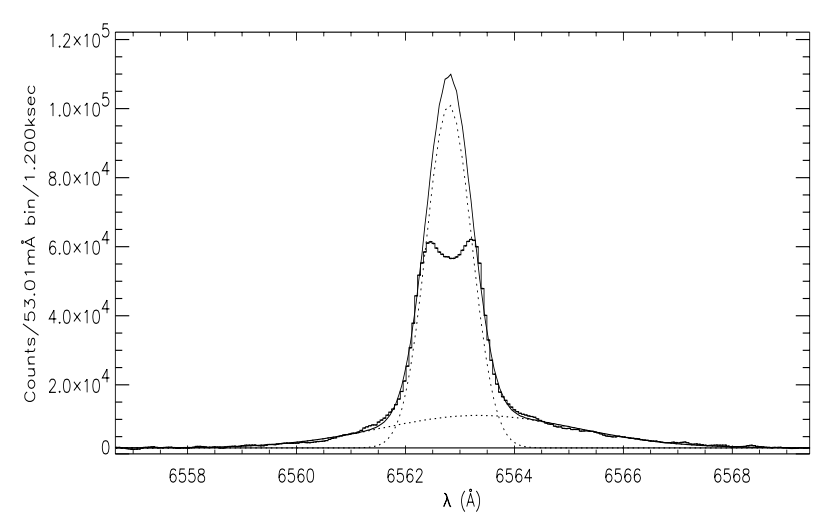

Fig. 6. Fit of the $\mathrm{H}_{\alpha}$ line with two components. The amplitude of the narrow component was fitted manually due to the severe selfabsorption. The fit parameters can be found in Table 3 for Spectrum 2.

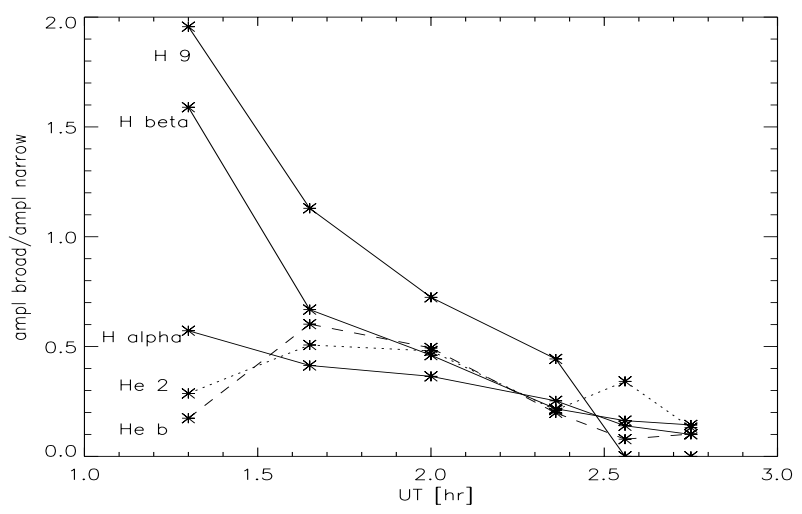

Fig. 7. Ratio of the amplitude of the broad line component to the narrow line component. The ratio between the main component of the $\mathrm{He} \mathrm{ID}_{3}$ line and its broad component is named "He b", and between the main component and the second component it is named "He 2".

constrained, and the errors of the $F W H M$, and especially the amplitude, are large. Fortunately, the fit parameters of the narrow component do not influence the fit parameters of the broad component too severely.

Using the procedure described above we fitted the $\mathrm{H}_{\alpha}, \mathrm{H}_{\beta}$, $\mathrm{H}_{9}, \mathrm{H}_{10}$, and $\mathrm{H}_{11}$ lines in the Balmer series and the He lines at 5875, 5015, 4921, 3819, and $3187 \AA$. Except for the first two spectra the He lines at 5015, 4921, 3819, and $3187 \AA$ required only a single line due to the low signal. This similarly applies to the high Balmer lines, where $\mathrm{H}_{9}$ and $\mathrm{H}_{10}$ lines show a broad component only in the first four spectra, while the $\mathrm{H}_{11}$ line shows a broad component only in the first three spectra. In the sixth spectrum for $\mathrm{H}_{9}$ the two-component fit leads to $\chi^{2}$ of 0.30 , while one component has a $\chi^{2}=0.31$. Therefore, two components do not lead to a substantial improvement of the fit. The fit parameters for the stronger lines are listed in Table 6 for He $\mathrm{ID}_{3}$, in Table 3 for the $\mathrm{H}_{\alpha}$ line, in Table 4 for the $\mathrm{H}_{\beta}$ line, and in Table 5 for the $\mathrm{H}_{9}$ line.

Fit amplitudes of both the narrow and the broad component decay with time, but the amplitude of the broad component decays much faster than that of the narrow component (cf., in Fig. 7). For the $\mathrm{He}_{\mathrm{I}} \mathrm{D}_{3}$ line the ratio increases from the first to the second spectrum and then decreases as for the Balmer lines. The same applies to the ratio between the main component of the $\mathrm{He} \mathrm{ID}_{3}$ line and its weaker component at $5875.97 \AA$. 
Table 3. Free fitting parameters for the $\mathrm{H}_{\alpha}$ line. The wavelength difference $\Delta \lambda$ between the narrow component and the broad component is also given. The amplitude of the narrow component is fitted manually and so no error estimate is given for this value. Since the peak of the narrow line was not included in the fitting process, no error computation of the central wavelength was carried out. We estimate the error to be similar to the values found for the other Balmer lines. Especially the value for the narrow component seems to be quite accurate compared to the rest wavelength of $6562.817 \AA$ of the line (Moore 1972).

\begin{tabular}{cccccccc}
\hline \hline $\mathrm{sp}$ & $\begin{array}{c}A_{\mathrm{n}} \\
{\left[10^{3} \text { electrons }\right]}\end{array}$ & $\begin{array}{c}A_{\mathrm{b}} \\
{\left[10^{3} \text { electrons }\right]}\end{array}$ & $\begin{array}{c}\sigma_{\mathrm{n}} \\
{[\AA]}\end{array}$ & $\begin{array}{c}\sigma_{\mathrm{b}} \\
{[\AA]}\end{array}$ & $\begin{array}{c}\lambda_{\mathrm{n}} \\
{[\AA]}\end{array}$ & $\begin{array}{c}\lambda_{\mathrm{b}} \\
{[\AA]}\end{array}$ & $\begin{array}{c}\Delta \lambda \\
{[\AA]}\end{array}$ \\
\hline 1 & 2150 & $1232 \pm 2$ & $0.40 \pm 0.04$ & $1.91 \pm 0.05$ & 6562.80 & 6562.81 & 0.01 \\
2 & 1950 & $807 \pm 1$ & $0.41 \pm 0.03$ & $1.78 \pm 0.01$ & 6562.78 & 6563.30 & 0.52 \\
3 & 1150 & $419 \pm 1$ & $0.40 \pm 0.04$ & $1.68 \pm 0.08$ & 6562.79 & 6563.65 & 0.86 \\
4 & 350 & $88 \pm 0.5$ & $0.39 \pm 0.04$ & $1.59 \pm 0.15$ & 6562.79 & 6563.55 & 0.76 \\
5 & 330 & $46 \pm 0.4$ & $0.36 \pm 0.03$ & $1.16 \pm 0.16$ & 6562.79 & 6563.28 & 0.49 \\
6 & 310 & $31 \pm 0.4$ & $0.35 \pm 0.04$ & 1.80 & 6562.79 & 6562.85 & 0.06 \\
7 & 510 & $130 \pm 0.7$ & $0.38 \pm 0.04$ & $1.62 \pm 0.16$ & 6562.82 & 6562.30 & -0.52 \\
8 & 810 & $319 \pm 0.9$ & $0.38 \pm 0.04$ & $1.76 \pm 0.02$ & 6562.82 & 6562.62 & -0.20 \\
\hline
\end{tabular}

Table 4. Fitting parameter for the $\mathrm{H}_{\beta}$ line. The given errors are formal ones and should be considered with caution. Sometimes no error estimates were possible. All given parameters besides $\Delta \lambda$ were free parameters in the fit. The rest wavelength of the $\mathrm{H}_{\beta}$ line is $4861.33 \AA$, showing that there are no wavelength shifts in the narrow component.

\begin{tabular}{cccccccc}
\hline \hline $\mathrm{sp}$ & $\begin{array}{c}A_{\mathrm{n}} \\
{\left[10^{3} \text { electrons }\right]}\end{array}$ & $\begin{array}{c}A_{\mathrm{b}} \\
{\left[10^{3} \text { electrons }\right]}\end{array}$ & $\begin{array}{c}\sigma_{\mathrm{n}} \\
{[\AA]}\end{array}$ & $\begin{array}{c}\sigma_{\mathrm{b}} \\
{[\AA]}\end{array}$ & $\begin{array}{c}\lambda_{\mathrm{n}} \\
{[\AA]}\end{array}$ & $\begin{array}{c}\lambda_{\mathrm{b}} \\
{[\AA]}\end{array}$ & $\begin{array}{c}\Delta \lambda \\
{[\AA]}\end{array}$ \\
\hline 1 & $608 \pm 1$ & $967 \pm 1$ & $0.37 \pm 0.02$ & 1.55 & $4861.32 \pm 0.01$ & $4861.39 \pm 0.01$ & $0.07 \pm 0.01$ \\
2 & $617 \pm 1$ & $412 \pm 1$ & 0.35 & 1.38 & 4861.32 & 4861.70 & 0.38 \\
3 & $311 \pm 1$ & $143 \pm 0.5$ & 0.30 & 1.26 & $4861.33 \pm 0.01$ & $4861.92 \pm 0.01$ & $0.59 \pm 0.01$ \\
4 & $80 \pm 0.3$ & $17 \pm 0.2$ & 0.26 & $0.94 \pm 0.03$ & $4861.33 \pm 0.01$ & $4861.78 \pm 0.01$ & $0.45 \pm 0.01$ \\
5 & $59 \pm 0.2$ & $9 \pm 0.2$ & $0.24 \pm 0.01$ & $0.92 \pm 0.02$ & $4861.32 \pm 0.08$ & $4861.75 \pm 0.04$ & $0.43 \pm 0.09$ \\
6 & $52 \pm 0.2$ & $7 \pm 0.2$ & 0.24 & $1.02 \pm 0.07$ & $4861.33 \pm 0.04$ & $4861.64 \pm 0.08$ & $0.31 \pm 0.09$ \\
7 & $74 \pm 0.3$ & $29 \pm 0.3$ & $0.27 \pm 0.01$ & 0.85 & $4861.34 \pm 0.04$ & $4861.23 \pm 0.01$ & $-0.11 \pm 0.04$ \\
8 & $143 \pm 0.5$ & $94 \pm 0.5$ & 0.29 & 0.98 & $4861.34 \pm 0.01$ & $4861.32 \pm 0.04$ & $-0.02 \pm 0.04$ \\
\hline
\end{tabular}

Table 5. Free fitting parameters for the $\mathrm{H}_{9}$ line. The wavelength difference $\Delta \lambda$ between the narrow component and the broad component is also given. In the first spectrum here are three and in the second spectrum two additional metal lines in the direct vicinity of $\mathrm{H}_{9}$ that had to be fitted simultaneously to give the correct line profile. Especially for the fitting of many lines, the errors should be treated with caution and should be considered only as formal errors.

\begin{tabular}{cccccccc}
\hline \hline $\mathrm{sp}$ & $\begin{array}{c}A_{\mathrm{n}} \\
\text { [electrons] }\end{array}$ & $\begin{array}{c}A_{\mathrm{b}} \\
{[\text { electrons] }}\end{array}$ & $\begin{array}{c}\sigma_{\mathrm{n}} \\
{[\AA]}\end{array}$ & $\begin{array}{c}\sigma_{\mathrm{b}} \\
{[\AA]}\end{array}$ & $\begin{array}{c}\lambda_{\mathrm{n}} \\
{[\AA]}\end{array}$ & $\begin{array}{c}\lambda_{\mathrm{b}} \\
{[\AA]}\end{array}$ & $\begin{array}{c}\Delta \lambda \\
{[\AA]}\end{array}$ \\
\hline 1 & $3316 \pm 84$ & $6489 \pm 127$ & $0.21 \pm 0.01$ & 1.15 & $3835.37 \pm 0.06$ & $3835.34 \pm 0.09$ & $-0.03 \pm 0.11$ \\
2 & $2520 \pm 66$ & $2846 \pm 83$ & $0.20 \pm 0.01$ & 1.00 & $3835.38 \pm 0.06$ & $3835.43 \pm 0.11$ & $0.05 \pm 0.13$ \\
3 & $671 \pm 41$ & $486 \pm 48$ & $0.16 \pm 0.01$ & $0.48 \pm 0.05$ & $3835.37 \pm 0.07$ & $3835.49 \pm 0.23$ & $0.12 \pm 0.24$ \\
4 & $124 \pm 19$ & $55 \pm 23$ & $0.14 \pm 0.01$ & 0.40 & $3835.38 \pm 0.08$ & $3835.45 \pm 0.27$ & $0.07 \pm 0.28$ \\
5 & $101 \pm 13$ & & $0.17 \pm 0.02$ & & $3835.37 \pm 0.16$ & & \\
6 & $75 \pm 11$ & & $0.14 \pm 0.01$ & & $3835.43 \pm 0.13$ & & \\
7 & $248 \pm 20$ & & $0.21 \pm 0.02$ & & $3835.40 \pm 0.14$ & & \\
8 & $458 \pm 32$ & $534 \pm 42$ & $0.19 \pm 0.02$ & $0.81 \pm 0.08$ & $3835.39 \pm 0.09$ & $3835.34 \pm 0.29$ & $-0.05 \pm 0.30$ \\
\hline
\end{tabular}

For the narrow components of all lines, Gaussian width $\sigma$ is almost constant or decreases only slowly. The broad component has a decreasing width $\sigma$ as well, but in the sixth spectrum it increases again slightly; we caution, however, that this may be an artifact from our fits, because the amplitude is very small for all lines and the true error in the fit parameters may be larger than indicated by the formal errors because of the fit's non-uniqueness.

The line shifts between the narrow and broad components vary differently from line to line as can be seen in Fig. 8 for different $\mathrm{He}$ and Balmer lines. While the Balmer lines have very similar line shifts in the first spectrum, their behavior deviates in the following spectra; the higher Balmer lines $\left(\mathrm{H}_{9}, \mathrm{H}_{10}\right.$, and $\mathrm{H}_{11}$ ) have a much smaller line shift in the broad component than the $\mathrm{H}_{\alpha}$ and $\mathrm{H}_{\beta}$ lines. In general, line shifts increase during the first three spectra and then start to decrease. For the He I lines, increase in the line shifts is observed only in the first

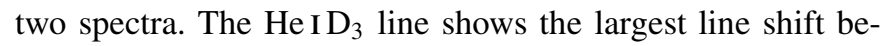
tween its narrow and broad component for all the lines in the second spectrum. 
Table 6. Fitting parameters for the $\mathrm{He}_{\mathrm{I}} \mathrm{D}_{3}$ line. In addition the wavelength difference $\Delta \lambda$ between the narrow component and the broad component is given. The two components of the multiplet have rest wavelength of 5875.62 and $5875.99 \AA$ showing the good quality of the multi-component fit.

\begin{tabular}{ccccccccrrr}
\hline \hline sp & $\begin{array}{c}A_{\mathrm{n}} \\
{\left[\mathrm{e}^{-}\right]}\end{array}$ & $\begin{array}{c}A_{\mathrm{s}} \\
{\left[\mathrm{e}^{-}\right]}\end{array}$ & $\begin{array}{c}A_{\mathrm{b}} \\
{\left[\mathrm{e}^{-}\right]}\end{array}$ & $\begin{array}{c}\sigma_{\mathrm{n}} \\
{[\AA]}\end{array}$ & $\begin{array}{c}\sigma_{\mathrm{s}} \\
{[\AA]}\end{array}$ & $\begin{array}{c}\sigma_{\mathrm{b}} \\
{[\AA]}\end{array}$ & $\begin{array}{c}\lambda_{\mathrm{n}} \\
{[\AA]}\end{array}$ & $\begin{array}{c}\lambda_{\mathrm{s}} \\
{[\AA]}\end{array}$ & $\begin{array}{c}\lambda_{\mathrm{b}} \\
{[\AA]}\end{array}$ & $\begin{array}{c}\Delta \lambda \\
{[\AA]}\end{array}$ \\
\hline 1 & $94178 \pm 361$ & $16357 \pm 325$ & $26978 \pm 254$ & 0.18 & 0.15 & $0.86 \pm 0.01$ & 5875.60 & 5875.97 & 5876.03 & 0.43 \\
2 & $59912 \pm 304$ & $30378 \pm 266$ & $36065 \pm 305$ & 0.17 & 0.20 & 1.26 & 5875.58 & 5875.93 & 5876.72 & 1.14 \\
3 & $24563 \pm 197$ & $11793 \pm 175$ & $12126 \pm 201$ & $0.16 \pm 0.01$ & $0.20 \pm 0.01$ & $1.10 \pm 0.03$ & $5875.59 \pm 0.01$ & 5875.93 & $5876.62 \pm 0.04$ & 1.03 \\
4 & $6698 \pm 94$ & $1411 \pm 60$ & $1326 \pm 95$ & $0.16 \pm 0.01$ & $0.13 \pm 0.01$ & $0.82 \pm 0.08$ & 5875.62 & $5876.00 \pm 0.02$ & $5876.52 \pm 0.14$ & 0.90 \\
5 & $3886 \pm 76$ & $1327 \pm 61$ & $304 \pm 46$ & 0.15 & $0.14 \pm 0.01$ & $0.22 \pm 0.02$ & $5875.59 \pm 0.01$ & $5875.93 \pm 0.02$ & $5876.28 \pm 0.08$ & 0.69 \\
6 & $3590 \pm 71$ & $470 \pm 46$ & $365 \pm 52$ & $0.17 \pm 0.01$ & $0.10 \pm 0.01$ & $0.26 \pm 0.03$ & $5875.61 \pm 0.01$ & $5875.98 \pm 0.03$ & $5876.24 \pm 0.07$ & 0.63 \\
7 & $7666 \pm 112$ & $2653 \pm 89$ & & 0.18 & 0.19 & & $5875.62 \pm 0.01$ & $5875.97 \pm 0.01$ & \\
8 & $15437 \pm 152$ & $5765 \pm 121$ & & 0.16 & 0.20 & & $5875.62 \pm 0.01$ & $5875.98 \pm 0.01$ & \\
\hline
\end{tabular}

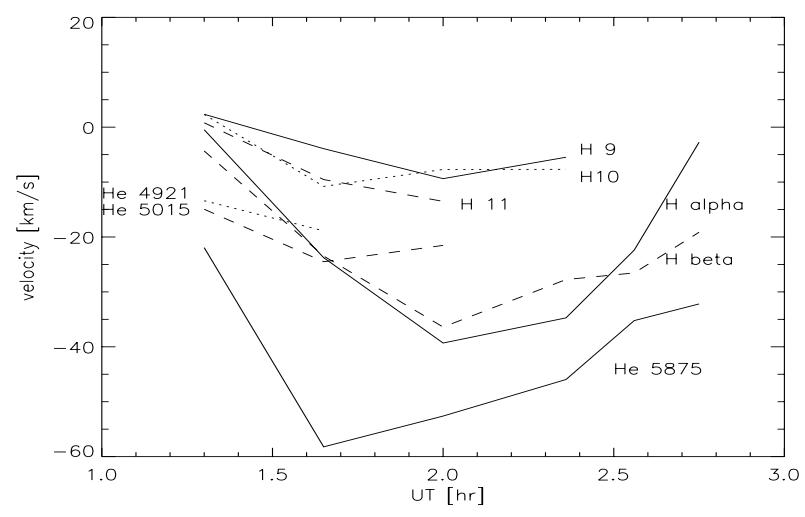

Fig. 8. Line shift between the narrow and the broad component of the different lines. Error should be typically about $10 \mathrm{~km} \mathrm{~s}^{-1}$.

\subsection{LHS 2034 in quiescence}

In addition to the spectra taken during the flare on March 14th, we obtained two additional spectra taken on March 16th. The photometer showed no obvious activity at that time; nevertheless, the emission lines vary in strength. The strength of the lines is rising from the seventh to the eighth spectrum again. The lines of the Balmer series again show a broad component with the amplitude a substantial fraction of the narrow component, or even exceeding it in the case of $\mathrm{H}_{9}$. The fitted central wavelength of the broad component is blueshifted for the $\mathrm{H}_{\alpha}$ and $\mathrm{H}_{\beta}$ lines for the seventh spectrum, whereas the fit of the $\mathrm{H}_{9}$ line is reasonably good without a broad component for this spectrum, as can be seen from Tables 3-5. In the last spectrum the $\mathrm{H}_{9}$ line again displays a broad line component but the line shift is consistent with zero, and the same applies to $\mathrm{H}_{\beta}$. Nevertheless the $\mathrm{H}_{\alpha}$ line seems to show a blueshift. Although the error of the central wavelengths could not be treated properly, it should be much smaller than the inferred wavelength shift. Table 6 shows that there is no broad component detected for the $\mathrm{He} \mathrm{ID}_{3}$ line in either of the two spectra.

\section{Chromosphere simulations of LHS 2034}

We compared our PHOENIX model chromosphere grid to the second spectrum taken during the flare where the largest asymmetries occur. This spectrum was taken in the decay phase of the flare, while the first spectrum contains part of the rise phase

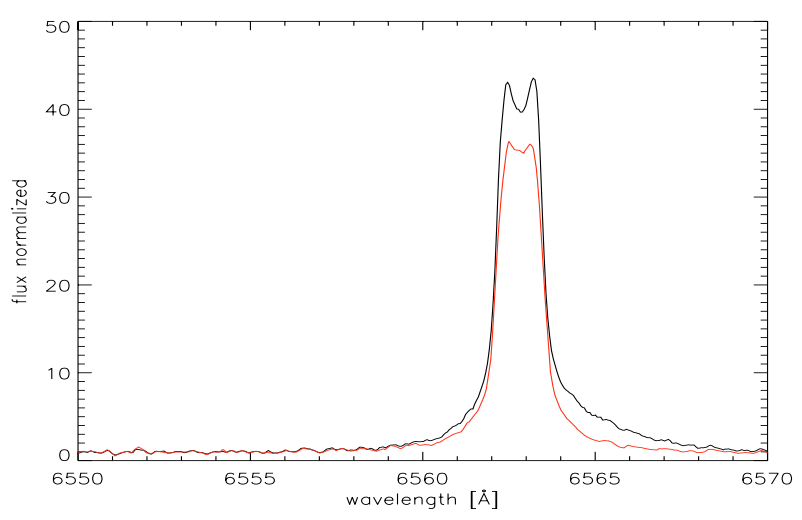

Fig. 9. Comparison of model (grey/red) and the first flare spectrum of LHS 2034 (black) around $\mathrm{H}_{\alpha}$.

of the flare. We used this second spectrum, since we computed all models in hydrostatic equilibrium, which is generally a poor approximation during the impulsive phase of a flare, but should not be too bad during the decay phase.

We use an $\chi^{2}$ algorithm to find the best fit model. Since our simulations suggested from the beginning that the flare would need a rather high filling factor, we take filling factors of 0.4 , $0.3,0.2,0.17,0.15,0.13,0.1,0.07,0.05,0.03$, and 0.01 into account. Indeed there are some indications that larger flares involve larger areas of the star rather than higher energy particle beams (Houdebine 2003).

The quality of the fit is checked for each filling factor using the following wavelength ranges: 6561.0 to $6562.8,4860.5$ to $4862.2,5870$ to 5900,3715 to 3755,3818 to 3830 , and 3840 to $3870 \AA$. The fit with the best mean $\chi^{2}$ for all wavelength ranges is indicated in Fig. 1 in black and has a filling factor of 20 percent. If the last spectrum in the flare series is not the one used as quiescent spectrum but instead the eighth spectrum taken on March 16th, then the best fit model has a transition region (TR) gradient 0.5 dex higher and a filling factor of 17 percent. Therefore the influence of the variations in the quiescent chromosphere is small. In Fig. 1 the two best fit models appear identical, because the difference in the TR gradient cannot be seen.

The quality of the fit can be seen for the $\mathrm{H}_{\alpha}$ line and around the Na D lines in Figs. 9 and 10 for the best overall model. The $\mathrm{Na} \mathrm{D}$ line emission is vastly underpredicted for all our models, 


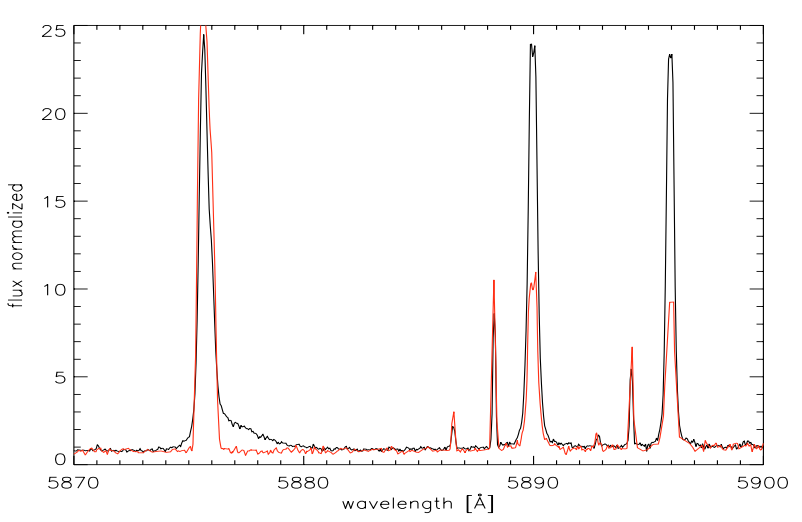

Fig. 10. Comparison of model (grey/red) and the first flare spectrum of LHS 2034 (black) around the $\mathrm{Na} \mathrm{D}$ lines and the $\mathrm{He}_{\mathrm{I}} \mathrm{D}_{3}$ line. While the He line is slightly overpredicted, the $\mathrm{Na} \mathrm{D}$ lines are vastly underpredicted. The narrow airglow lines are found in the model since we used a quiescent observed spectrum to mix with.

although our grid contains models with different temperature distributions in the lower chromosphere to which the $\mathrm{Na} D$ line emission is sensitive. Many of the Fe lines in the blue part of the spectrum are underpredicted as well, while the high Balmer lines in the same wavelength regime and the $\mathrm{H}_{\beta}$ line are overpredicted.

We investigated the approximate line formation depth of individual lines using spectra of each layer of the model atmosphere. Formation of the resulting spectrum in the outermost layer can be observed from layer to layer. One problem with this ansatz is that the net flux is tracked, i.e. the flux going inward is accounted for as well. We inferred from these layer spectra that the $\mathrm{He}$ and $\mathrm{H}$ lines are formed in the upper chromosphere, while the $\mathrm{Na} \mathrm{D}$ lines and the wings of $\mathrm{Fe} \mathrm{I}$ and Fe II and $\mathrm{Mg}$ I lines are formed in the middle and lower chromosphere. Therefore the phenomenon that only the $\mathrm{H}$ and $\mathrm{He}$ lines are showing line asymmetries is explained by the different formation depth of the lines. Accordingly the observed downflows must originate in a restricted area in the upper chromosphere or at least in material with similar temperatures.

Because the measured $F W H M$ of the broad redshifted components of the emission lines may be interpreted as turbulent velocity, we compared the inferred turbulent velocities of about 80 to $90 \mathrm{~km} \mathrm{~s}^{-1}$ with the sound velocity in the upper chromosphere as given by the models, which is about $10 \mathrm{~km} \mathrm{~s}^{-1}$. This indicates that the FWHM is not caused by microturbulence but that the broad feature may actually consist of more than one condensation moving at super sonic velocity with respect to their surroundings.

Moreover we investigated the behaviour of multiplet components of the $\mathrm{He} \mathrm{ID}_{3}$ line. The relative height both of the weaker component and the main component can be explained either by changes in the gradient of the TR or by changes in the temperature at the onset of the TR. Higher gradients and lower pressure at the onset of the TR result in a lower relative amplitude of the multiplet component at $5875.97 \AA$ compared

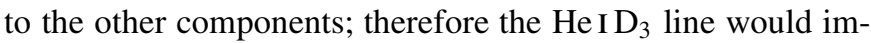
ply that the gradient of the TR should be increased by about 1 dex for the highest pressure models. Temperature at the top

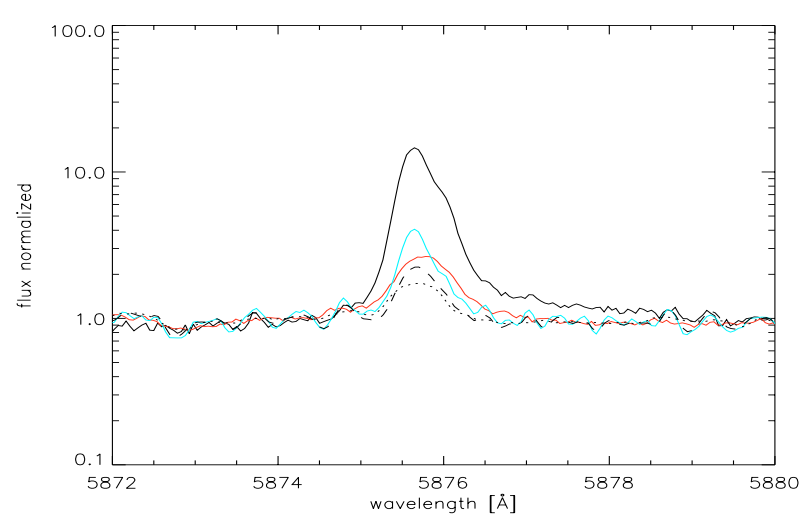

Fig. 11. Comparison of the $\mathrm{He}_{\mathrm{I}} \mathrm{D}_{3}$ line for a number of program stars. The black line denotes LHS 2034 where the asymmetry can be seen clearly. Also plotted are the flare spectrum of LHS 2076 (grey/red), the strongest CN Leo spectrum (light grey/turquoise), and the averaged spectrum of GL Vir (dotted) and of YZ Cmi (dashed). No strong wing asymmetries are found in the other spectra, except the bump that originates from the reddest line component.

of the chromosphere has less influence on the ratio of the com-

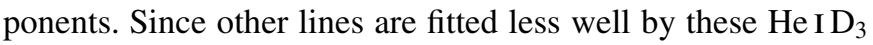
defined models we could not find models that fit many lines equally well.

\section{Discussion and conclusions}

\subsection{Asymmetries in other program stars?}

The asymmetries seen in LHS 2034 are unique in their strength for the 21 late-type $\mathrm{M}$ and $\mathrm{L}$ dwarfs of our program originally designed to search for the forbidden Fe XIII line at $3388 \AA$. The spectral characteristics for the mid-type $\mathrm{M}$ dwarfs are discussed in Fuhrmeister et al. (2004b) and for the whole sample in Fuhrmeister et al. (2004a). During the observations short duration flares also occurred on LHS 2076 and CN Leo. For $\mathrm{CN}$ Leo there is, in addition, a one-hour observation showing enhanced line flux, while the photometer does not show

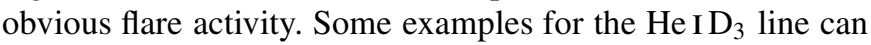
be found in Fig. 11 comparing it to LHS 2034. A similar line asymmetry could be found in none of the program stars, except LHS 2397a where very weak red asymmetries were found. For $\mathrm{CN}$ Leo we checked if a broad component might be present without being recognized due to low $S / N$ ratio. We took the $\mathrm{CN}$ Leo spectrum and measured the amplitude of the (narrow) $\mathrm{He} \mathrm{I} \mathrm{D}_{3}$ line at about 260000 electrons. For LHS 2034 the amplitude of the broad component is about half that of the narrow component. Therefore we took about 100000 electrons and added them to the $\mathrm{CN}$ Leo spectrum in a broad component with $\sigma=0.9 \AA$ and central wavelength at 5876.1 $\AA$ adding Poisson noise. The resulting feature was easily recognizable. Since all mid-type $M$ dwarfs in the sample have $S / N$ ratios similar to $\mathrm{CN}$ Leo, we conclude that no other star is exhibiting strong asymmetries as observed in LHS 2034. 


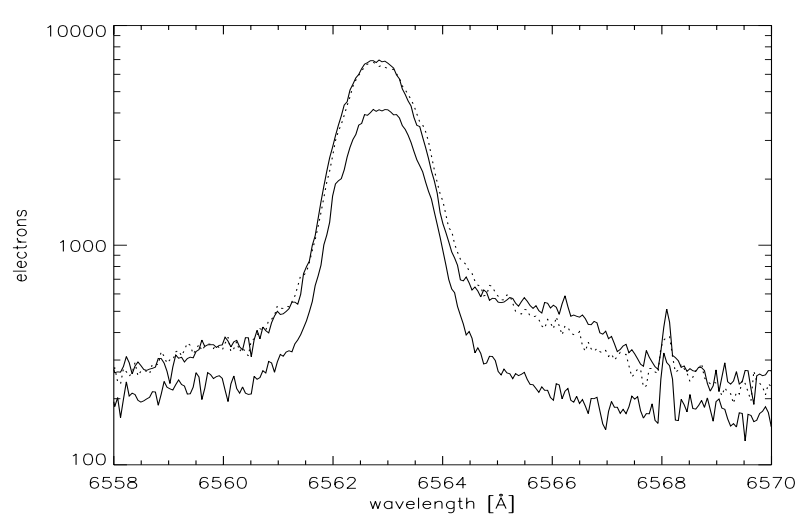

Fig. 12. The $\mathrm{H}_{\alpha}$ line for LHS 2397a. While the first two spectra show enhanced $\mathrm{H}_{\alpha}$ flux and some red wing asymmetries, the third spectrum fits well with only one component.

\subsection{LHS 2397a}

As mentioned above weak asymmetries were also found for LHS 2397a. LHS 2397a (GJ 3655) is a dM8 flare star that was recently discovered to have a brown dwarf companion of spectral type L7.5 (Freed et al. 2003). LHS 2397a shows a large spread in its recorded $\mathrm{H}_{\alpha}$ equivalent widths ranging from 15.3 to $47.3 \AA$ (Tinney \& Reid 1998; Gizis et al. 2000). We have three spectra of LHS 2397a resulting in one-hour total exposure. In two of the three spectra of the combined light from LHS 2397a and the L dwarf LHS 2397b, line asymmetries are seen; since no lines appear to be double we assume in the following that the signal is dominated by LHS 2397a. The photometer shows that the star was quiescent during the observations. Nevertheless, LHS 2397a shows very strong $\mathrm{H}_{\alpha}$ and $\mathrm{H}_{\beta}$ emission with an $E W$ of about $57 \AA$ for the $\mathrm{H}_{\alpha}$ line and $38 \AA$ for the $\mathrm{H}_{\beta}$ line in the first two spectra. In the last spectrum the $\mathrm{H}_{\alpha}$ emission line has decreased to an $E W$ of $33 \AA$. While the $\mathrm{H}_{\alpha} E W$ for the first two spectra is considerably higher than the range of 15.3 to $47.3 \AA$ found by Tinney \& Reid (1998) and Gizis et al. (2000), the $E W$ found in the last spectrum seems to be quite normal for LHS 2397a. Both the $\mathrm{H}_{\alpha}$ and $\mathrm{H}_{\beta}$ line are observed to have a broad component that is redshifted with respect to the narrow component. The line asymmetries disappear in the last spectrum. The asymmetries are shown in Fig. 12. LHS 2397a also shows a rather weak $\mathrm{He}_{\mathrm{ID}} \mathrm{D}_{3}$ line and the Balmer series up to $\mathrm{H}_{11}$ thus providing more evidence for its high activity even during apparent quiescence. Because of the fairly poor signal to noise ratios in these lines we cannot demonstrate the existence of a broad component.

The free fit parameters for the $\mathrm{H}_{\alpha}$ and $\mathrm{H}_{\beta}$ lines are given in Table 7. The ratio of amplitude between the broad and the narrow components is for LHS 2397a in the same range as for LHS 2034 at the end of the flare in the fourth, fifth, and sixth spectra. The width of the narrow line component is consistent with rotational broadening, so like LHS 2034 the line seems to originate in not just one single active region. The width of the broad component is about the same value as the largest width found for LHS 2034.

Little can be said about the relative velocities between the narrow and broad components since the measurements of the
$\mathrm{H}_{\beta}$ line have large errors, and the broad line is very shallow. For the $\mathrm{H}_{\alpha}$ line the velocity is decelerated from 49 to $26 \mathrm{~km} \mathrm{~s}^{-1}$. Measurements for the $\mathrm{H}_{\beta}$ line are consistent with these measurements for $\mathrm{H}_{\alpha}$, as well as with the hypothesis that the velocity between narrow and broad component of $\mathrm{H}_{\beta}$ does not change at all for the two spectra.

An origin of the additional emission from the secondary L dwarf can be ruled out. Since the estimated orbital period is about 25 years (Freed et al. 2003) and the separation is $2.96 \mathrm{AU}$, the expected maximum velocity shift for lines from the secondary is about $3.5 \mathrm{~km} \mathrm{~s}^{-1}$ in contrast to the about $30 \mathrm{~km} \mathrm{~s}^{-1}$ observed for LHS 2397a. Since the $E W$ of the $\mathrm{H}_{\alpha}$ and $\mathrm{H}_{\beta}$ line is rather high in the first two spectra, the asymmetries may nevertheless be connected with activity. Simultaneously taken X-ray data would help to better understand the activity connection of the asymmetries, once more pointing out the need for multi-wavelength observations of flare connected phenomena. As it stands now the case of LHS 2397a is rather confusing since the photometer diagnostic contrasts with the line diagnostic of the equivalent width. Mass motions are not a likely explanation of the broad component since they are only expected in flares. Another explanation in the case of LHS 2397a is additional emission from an active region since the inferred maximum velocity is about $30 \mathrm{~km} \mathrm{~s}^{-1}$. Again considering the errors of about $10 \mathrm{~km} \mathrm{~s}^{-1}$ this is in agreement with the rotational velocity of LHS 2397a. Mohanty \& Basri found LHS 2397a to be a fast rotator with a $v \sin (i)$ of $20 \mathrm{~km} \mathrm{~s}^{-1}$. As long as we lack a good template star we cannot measure its rotational velocity; however, the $F W H M$ of the emission lines of LHS 2397a is consistent with rotational velocity of 20 to $30 \mathrm{~km} \mathrm{~s}^{-1}$. Therefore, flickering in an active region near the limb of LHS 2397a may cause the broad component without leading to a flare signature in the photometer data.

\subsection{LHS 2034}

Since we also do not see line asymmetries in any of the models, the observed asymmetries should not be an intrinsic part of the line profile. We therefore attribute the additional red wing flux in the Balmer and He lines to Doppler shifted emission from downward moving material. An alternative to emitting down-moving material would be an up-moving absorption layer. Then the Balmer and He lines would have to be symmetrically Stark broadened and the blue wing would be absorbed, leaving the red wing asymmetry. There are two arguments against that. First, we did not see symmetrically broadened lines in any of our chromospheric models. And second, the asymmetries are fitted quite well with two Gaussian emission lines. If they were instead composed of one emission and one absorption line, we would expect a poor fit with two emission lines especially in the blue wing.

Since we do see similar asymmetries in LHS 2397a without a flare, the asymmetries in LHS 2034 and the flare may be pure coincidence and not physically connected. The broad emission feature may then originate in a cloud co-rotating with the star as was proposed for transient absorption features in the K0 star AB Dor by Collier Cameron \& Robinson (1989). In this case 
Table 7. Free fitting parameters for LHS 2397a for the $\mathrm{H}_{\alpha}$ (top) and the $\mathrm{H}_{\beta}$ (bottom) line. In addition the wavelength difference $\Delta \lambda$ between the narrow component and the broad component is given.

\begin{tabular}{cccccccc}
\hline \hline sp & $\begin{array}{c}A_{\mathrm{n}} \\
{\left[10^{3} \text { electrons }\right]}\end{array}$ & $\begin{array}{c}A_{\mathrm{b}} \\
{\left[10^{3} \text { electrons }\right]}\end{array}$ & $\begin{array}{c}\sigma_{\mathrm{n}} \\
{[\AA]}\end{array}$ & $\begin{array}{c}\sigma_{\mathrm{b}} \\
{[\AA]}\end{array}$ & $\begin{array}{c}\lambda_{\mathrm{n}} \\
{[\AA]}\end{array}$ & $\begin{array}{c}\lambda_{\mathrm{b}} \\
{[\AA]}\end{array}$ & $\begin{array}{c}\Delta \lambda \\
{[\AA]}\end{array}$ \\
\hline 1 & $173 \pm 0.5$ & $34 \pm 0.4$ & $0.56 \pm 0.01$ & $2.04 \pm 0.01$ & $6562.80 \pm 0.01$ & $6563.87 \pm 0.01$ & $0.07 \pm 0.01$ \\
2 & $169 \pm 0.5$ & $38 \pm 0.4$ & $0.57 \pm 0.01$ & 1.88 & $6562.85 \pm 0.01$ & $6563.42 \pm 0.21$ & $0.57 \pm 0.21$ \\
3 & $115 \pm 0.4$ & \pm 0.61 & 0.06 & & $6562.88 \pm 0.33$ & & \\
\hline 1 & $33 \pm 0.2$ & $7 \pm 0.2$ & $0.35 \pm 0.01$ & $1.32 \pm 0.07$ & $4861.34 \pm 0.05$ & $4861.93 \pm 0.37$ & $0.59 \pm 0.37$ \\
2 & $35 \pm 0.2$ & $12 \pm 0.2$ & 0.37 & $1.43 \pm 0.04$ & $4861.39 \pm 0.10$ & $4861.90 \pm 0.05$ & $0.51 \pm 0.11$ \\
3 & $20 \pm 0.2$ & & $0.39 \pm 0.02$ & & $4861.43 \pm 0.15$ & & \\
\hline
\end{tabular}

the Doppler shift is not caused by a downward motion but by the rotation of the co-rotating cloud that is about to set behind the star. By assuming of an inclination close to $90^{\circ}$ and the cloud close to the equatorial plane, one can compute the distance of the cloud from the star's surface as $d=\frac{v P}{2 \pi}$ with $P$ the rotation period of the star and $v$ the radial velocity of the cloud. With an estimated radius of the star of $R_{*}=0.1 R_{\odot}$ and measured $v \sin i=8 \mathrm{~km} \mathrm{~s}^{-1}$ one obtains $P=15 \mathrm{~h}$, and with a radial velocity of the Balmer line feature of about $30 \mathrm{~km} \mathrm{~s}^{-1}$ one obtains a distance of $d=3.9 R_{*}$. Variation in the cloud's radial velocity would then be due to the geometry with peak velocity reached when the cloud is besides the star and diminishing while the cloud rotates behind the star. Since the distance to the star is fairly high, the cloud need not to rotate behind the star during about $1 \mathrm{~h}$ of diminishing radial velocities. But if the cloud is not obscured by the star in this scenario, the monotonic decreasing line strength is hard to explain. Another problem is the large $F W H M$ that leads to supersonic turbulent velocities if the cloud is at chromospheric temperatures and in hydrostatic equilibrium.

A solution to both problems may be a co-rotating cloud that has become unstable during the onset of the flare and is now expanding and cooling. But since the FWHM of the broad emission features seen in LHS 2397a are equivalent and no obvious flare activity was noticed there, we regard this scenario as unlikely. And since the strength of the broad emission lines is decaying even faster than the Balmer and He lines, a stronger connection between the excess emission seen in LHS 2034 and the flare is suggested.

One explanation for the asymmetries may be a magnetically confined cloud that has lost its supporting magnetic field during the flare and is falling down onto the star, with the deceleration caused by penetration of denser layers in the atmosphere of the star. The decay in amplitude would then be interpreted as cooling of the cloud; however, with an averaged velocity of about $30 \mathrm{~km} \mathrm{~s}^{-1}$ the cloud would be able to fall about $2.3 R_{\star}$ (assuming the radius of the star to be $0.1 R_{\odot}$ ). This seems to be a rather odd place for the origin of a magnetically confined cloud. Moreover, such a scenario could not explain the restriction of the asymmetries to the $\mathrm{He}$ and $\mathrm{H}$ lines since a falling cloud should have a broad temperature distribution.

Another explanation of the asymmetries are mass flows similar to chromospheric downward condensations (CDC) as known for the Sun (Canfield et al. 1990). While the downflows found during a flare in AD Leo (Houdebine et al. 1993) had velocities of up to $800 \mathrm{~km} \mathrm{~s}^{-1}$ our downflows have peak velocities of up to $60 \mathrm{~km} \mathrm{~s}^{-1}$, which is in the velocity range of CDCs on the Sun (e.g. Fisher 1989). Fisher (1989) also shows that the peak downflow velocity is related to the total flare energy flux at the peak of the flare $F_{\text {evap }}$ as follows:

$v_{\text {peak }} \sim 0.4\left(\frac{F_{\text {evap }}}{\rho}\right)^{1 / 3}$

with $\rho$ the chromospheric density. We estimate $F_{\text {evap }}$ by totalling the flux in our first spectrum, converting to the surface flux using $R=0.1 R_{\odot}$ and $d=13.9 \mathrm{pc}$, and dividing by the filling factor. We then obtain $F_{\text {evap }}=2.1 \times 10^{10} \mathrm{erg} \mathrm{cm}^{-2} \mathrm{~s}^{-1}$ and $\rho=3.8 \times 10^{-10} \mathrm{~g} \mathrm{~cm}^{-3}$ from our flare model for the upper chromosphere leading to peak velocities of about $15 \mathrm{~km} \mathrm{~s}^{-1}$. Our observed velocities tend to be smaller than this value except for the $\mathrm{He} \mathrm{ID}_{3}$ line. Measured velocities for the first spectrum tend to be lower but are in agreement with this value, considering the rather large errors (see Fig. 8).

For the Sun such events can last a few minutes, whereas the asymmetries on LHS 2034 last for $1.5 \mathrm{~h}$. Since the chromospheric modelling gives strong evidence that mass motions occur in the upper part of the chromosphere, a series of downward moving condensations is needed. The FWHM of the broad components compared to the sound velocity also indicates that there is more than one condensation present at the same time. Thus the favourite scenario of the asymmetries is a series of flare-triggered downward moving condensations in the upper chromosphere. Since our spectra have rather long integration times compared to the lifetime of condensations on the Sun, condensations occurring at the same time at different places in the flare region cannot be distinguished from condensations occurring at the same place during the integration time.

\subsection{Summary}

In our VLT spectra of two late-type M dwarfs we find evidence for downward directed mass motions manifesting themselves in red wing asymmetries of hydrogen and helium lines. Surprisingly, both stars belong kinematically to the old disk despite their rather high level of activity during our observations. Chromospheric modeling with the atmosphere code PHOENIX suggests that the lines showing downward motions are formed in the high chromosphere. For LHS 2034, these chromospheric flare models lead to fairly high filling factors of about 20 percent for the flaring chromosphere. Furthermore, the width of the broad emission feature building up the line asymmetry is unlikely to be due to turbulent velocity. This would 
require supersonic velocities in the upper chromosphere, so we prefer interpreting it as a series of downflows.

Acknowledgements. The model computations were performed at the Norddeutscher Verbund für Hoch- und Höchstleistungsrechnen (HLRN) and at the Hamburger Sternwarte Apple G5 cluster financially supported by HBFG. We thank Dr. R. Wichmann for the computation of the space velocities. B.F. acknowledges financial support by the Deutsche Forschungsgemeinschaft under DFG SCHM 1032/16-1. P.H.H. was supported in part by the Pôle Scientifique de Modélisation Numérique at ENS-Lyon.

\section{References}

Canfield, R. C., Penn, M. J., Wulser, J., \& Kiplinger, A. L. 1990, ApJ, 363,318

Collier Cameron, A., \& Robinson, R. D. 1989, MNRAS, 236, 57

Doyle, J. G., Butler, C. J., Bryne, P. B., \& van den Oord, G. H. J. 1988, A\&A, 193, 229

Favata, F., Reale, F., Micela, G., et al. 2000, A\&A, 353, 987

Fisher, G. H. 1989, ApJ, 346, 1019

Fleming, T. A., Giampapa, M. S., Schmitt, J. H. M. M., \& Bookbinder, J. A. 1993, ApJ, 410, 387

Freed, M., Close, L. M., \& Siegler, N. 2003, ApJ, 584, 453

Fuhrmeister, B., Schmitt, J. H. M. M., \& Hauschildt, P. H. 2004a, A\&A, submitted
Fuhrmeister, B., Schmitt, J. H. M. M., \& Wichmann, R. 2004b, A\&A, 417, 701

Gizis, J. E., Monet, D. G., Reid, I. N., et al. 2000, AJ, 120, 1085

Gunn, A. G., Doyle, J. G., Mathioudakis, M., Houdebine, E. R., \& Avgoloupis, S. 1994, A\&A, 285, 489

Hauschildt, P. H., Allard, F., \& Baron, E. 1999, ApJ, 512, 377

Houdebine, E. R. 2003, A\&A, 397, 1019

Houdebine, E. R., Foing, B. H., Doyle, J. G., \& Rodono, M. 1993, A\&A, 274, 245

Kürster, M., \& Schmitt, J. H. M. M. 1996, A\&A, 311, 211

Kurucz, R. L., \& Bell, B. 1995, Atomic line list Kurucz CD-ROM, Cambridge, MA: Smithsonian Astrophysical Observatory, April 15,1995

Leggett, S. K. 1992, ApJS, 82, 351

Mauas, P. J. D., \& Falchi, A. 1996, A\&A, 310, 245

Mohanty, S., \& Basri, G. 2003, ApJ, 583, 451

Montes, D., Saar, S. H., Collier Cameron, A., \& Unruh, Y. C. 1999, MNRAS, 305, 45

Moore, C. E. 1972, Nat. Stand. Ref. Data Ser., 40

Ness, J.-U., \& Wichmann, R. 2002, Astron. Nachr., 323, 129

Pagano, I., Linsky, J. L., Carkner, L., et al. 2000, ApJ, 532, 497

Pettersen, B. R. 1991, Mem. della Soc. Astron. Ital., 62, 217

Preibisch, T., Neuhaeuser, R., \& Alcala, J. M. 1995, A\&A, 304, L13

Reid, I. N., Hawley, S. L., \& Gizis, J. E. 1995, AJ, 110, 1838

Schmitt, J. H. M. M., \& Favata, F. 1999, Nature, 401, 44

Tinney, C. G., \& Reid, I. N. 1998, MNRAS, 301, 1031

Young, P. R., Del Zanna, G., Landi, E., et al. 2003, ApJS, 144, 135 